\title{
Efeito do treinamento pliométrico e isométrico na força explosiva de atletas de handebol
}

\author{
Effect of plyometric and isometric training on the explosive force \\ of handball athletes
}

\section{Efecto del entrenamiento pliométrico e isométrico en la fuerza explosiva de los atletas de balonmano}

\author{
*João Victor da Costa Alecrim, ** Jose Victor da Costa Alecrim Neto, \\ *** Mariana Oliveira Souza, ${ }^{* * *}$ Gilberto Pivetta Pires
}

\begin{abstract}
da Costa Alecrim, J., da Costa Alecrim Neto, J., Oliveira, M., Pivetta, G. (2019). Efeito do treinamento pliométrico e isométrico na força explosiva de atletas de handebol. Revista Ciencias de la Actividad Física UCM, № 20(2), juliodiciembre, 1-14. DOI: http://doi.org/10.29035/rcaf.20.2.10
\end{abstract}

\begin{abstract}
RESUMO
A pliometria utiliza a capacidade reativa do sistema neuromuscular para aumentar a potência do movimento partindo de uma contração excêntrica para uma concêntrica. A isometria refere-se a uma modalidade estática de treinamento, ou seja, não há alteração no comprimento da fibra muscular. O handebol é uma modalidade esportiva coletiva que envolve uma grande quantidade e variedade de movimentação associada à manipulação de bola e interação com outros atletas. Em termos de movimentação, o handebol pode ser considerado um esporte completo, pois utiliza uma rica combinação das habilidades motoras fundamentais e "naturais" do repertório motor do ser humano. O objetivo da pesquisa é analisar a influência de 4 semanas de treinamento pliométrico e isométrico na força explosiva de jovens atletas de handebol. A amostra contou com 10 mulheres, com média de idade de 16,1 \pm 0,56 anos, praticantes de handebol há pelo menos três meses. O protocolo de avaliação utilizado foi: salto horizontal (SH) para mensurar força explosiva de membro inferior. As meninas foram divididas em dois grupos estatisticamente iguais, o grupo pliométrico (GP) e o isométrico (GI). Os dados foram analisados através de teste-t pareado e Effect Size (magnitude de efeito), além do manual Proesp. Inicialmente os testes apresentaram os seguintes resultados: o GP mostrou 1,32 $\pm 0,13 \mathrm{~m}$ no $\mathrm{SH}$ e o $\mathrm{Gl} 1,41 \pm 0,10 \mathrm{~m}$. Após quatro semanas de treinamento foram refeitos os testes e o GP evidenciou 1,37 $\pm 0,08 \mathrm{~m}$ no $\mathrm{SH}$ e o Gl 1,43 $\pm 0,09 \mathrm{~m}$. Estatisticamente as mudanças não se apresentaram significativas, a magnitude do efeito os resultados de força explosiva de membros inferiores mostraram-se superficiais. Conclui-se então que apesar de estatisticamente insignificantes, ambos os métodos apresentam pequena evolução na força explosiva de membros inferiores, e cabe analisar maior tempo dos métodos nesses ganhos e também analisar uma proposta concomitante entre ambos os métodos
\end{abstract}

\section{PALAVRAS-CHAVE}

Força, Pliometria, Isometria, Membros Inferioires.

\footnotetext{
* Laboratório de Biociência e Motricidade Humana de Roraima. Boa Vista, Brasil.

** Centro Universitário Internacional - UNINTER. Boa Vista, Brasil.

***Instituto Federal de Roraima - IFRR. Boa Vista, Brasil.
} 
da Costa Alecrim, J., da Costa Alecrim Neto, J., Oliveira, M., Pivetta, G. (2019). Efeito do treinamento pliométrico e isométrico na força explosiva de atletas de handebol. Revista Ciencias de la Actividad Física UCM, N²0(2), julio-diciembre, 1-14. DOI: http://doi.org/10.29035/rcaf.20.2.10

\begin{abstract}
Plyometrics uses the reactive capacity of the neuromuscular system to increase the power of movement from an eccentric to a concentric contraction. Isometry refers to a static mode of training, ie there is no change in muscle fiber length. Handball is a collective sport that involves a large amount and variety of movement associated with ball manipulation and interaction with other athletes. In terms of movement, handball can be considered a complete sport because it uses a rich combination of fundamental and "natural" motor skills of the human motor repertoire. The aim of this research is to analyze the influence of a 4 week plyometric and isometric training on the explosive strength of young handball athletes. The sample consisted of 10 women, with an average age of $16.1 \pm 0.56$ years, who had been practicing handball for at least three months. The evaluation protocol used was: horizontal jump (HS) to measure lower limb explosive strength. The girls were divided into two statistically equal groups, the plyometric group (GP) and the isometric group (GI). Data were analyzed using paired t-test and Effect Size, as well as the Proesp manual. Initially the tests presented the following results: GP showed $1.32 \pm 0.13 \mathrm{~m}$ in $\mathrm{HS}$ and $\mathrm{Gl} 1.41 \pm 0.10 \mathrm{~m}$. After four weeks of training, the tests were redone and the GP showed $1.37 \pm 0.08 \mathrm{~m}$ in $\mathrm{HS}$ and the Gl $1.43 \pm 0.09 \mathrm{~m}$. Statistically the changes were not significant, the magnitude of the effect and the lower explosive strength results were superficial. It is concluded that, although statistically insignificant, both methods present little evolution in the explosive force of lower limbs, and it is necessary to further analyze the methods in these gains and also to analyze the concurrent proposal between both methods.
\end{abstract}

\title{
Key words
}

Strength, Plyometrics, Isometry, Inferior limbs.

\section{RESUMEN}

La pliometría utiliza la capacidad reactiva del sistema neuromuscular para aumentar el poder de movimiento de una contracción excéntrica a una concéntrica. La isometría se refiere a un modo estático de entrenamiento, es decir, no hay cambios en la longitud de la fibra muscular. El balonmano es un deporte colectivo que involucra una gran cantidad y variedad de movimientos asociados con la manipulación del balón y la interacción con otros atletas. En términos de movimiento, el balonmano puede considerarse un deporte completo porque utiliza una rica combinación de las habilidades motoras fundamentales y "naturales" del repertorio motor humano. El objetivo de esta investigación es analizar la influencia de 4 semanas de entrenamiento pliométrico e isométrico en la fuerza explosiva de los jóvenes atletas de balonmano. La muestra consistió en 10 mujeres, con una edad promedio de 16.1 土 0.56 años, que habían practicado balonmano durante al menos tres meses. El protocolo de evaluación utilizado fue: salto horizontal (HS) para medir la fuerza explosiva de la extremidad inferior. Las niñas se dividieron en dos grupos estadísticamente iguales, el grupo pliométrico (GP) y el grupo isométrico (GI). Los datos se analizaron usando la prueba t pareada y el Tamaño del efecto, así como el manual Proesp. Inicialmente, las pruebas presentaron los siguientes resultados: GP mostró $1.32 \pm 0.13 \mathrm{~m}$ en HS y Gl $1.41 \pm 0.10 \mathrm{~m}$. Después de cuatro semanas de entrenamiento, las pruebas se rehicieron y el GP mostró $1.37 \pm 0.08 \mathrm{~m}$ en HS y el Gl $1.43 \pm 0.09 \mathrm{~m}$. Estadísticamente, los cambios no fueron significativos, la magnitud del efecto y los resultados de menor fuerza explosiva fueron superficiales. Se concluye que, aunque estadísticamente insignificante, ambos métodos presentan poca evolución en la fuerza explosiva de las extremidades inferiores, y es necesario analizar un mayor tiempo de los métodos en estas ganancias y también analizar una propuesta concurrente entre ambos métodos.

\section{Palabras clave}

Fuerza, pliometría, isometría, extremidades inferiores. 


\section{INTRODUÇÃO}

handebol é um esporte coletivo que proporciona uma variedade de movimentos, conexa à manipulação de bola e interação com a equipe. Toda essa movimentação é executada em quadra, cujas dimensões oficiais são 40 metros de comprimento e 20 metros de largura, com uma área de gol em cada extremidade, com 6 metros a partir da linha de fundo. Os esportes coletivos de quadra são caracterizados por esforços de alta intensidade e curta duração, apresentando movimentos de caráter anaeróbio (Kokubon e Daniel, 1992). O handebol, quando se trata de movimentação, pode ser considerado um esporte completo, pois faz uso de uma grande combinação de habilidades motoras "naturais" do repertório motor do humano (Martini, 1980). Durante uma partida de modalidade coletiva, principalmente em espaços reduzidos, ocorre mudança de períodos de esforço e recuperação. Essa alternância entre esforço e pausa se assemelha às necessidades do método de treinamento intermitente. Através do trabalho intermitente, é possível treinar as três vias energéticas, pois estão ligadas e atuam simultaneamente durante a atividade, suprindo a necessidade do indivíduo. Segundo Mais e Santos (apud Eleno, Barelo e Kokubun, 2002) a movimentação dos jogadores de handebol é variada apresentando movimentações em todas as intensidades, desde alta até a baixa. É muito frequente a realização de sprints, durante retorno para a defesa, ou contra-ataques, arremessos e movimentações de jogadas mais ofensivas, sendo esses caracterizados como movimentos de alta intensidade. $\bigcirc$ handebol necessita de força, velocidade, resistência, flexibilidade, coordenação e equilíbrio, além das variedades formadas por sua combinação, essas atividades são de extrema importância dentro de uma partida podendo ser decisivas para o resultado, por isso, essas valências também devem ser trabalhadas nos treinamentos, para que os movimentos se tornem mais precisos e energeticamente econômicos, resultando em melhor rendimento e desempenho dos atletas durante o jogo.

Faigenbaum et al. (2005) diz que o treinamento resistido refere-se a um método especializado de condicionamento, pelo qual - aluno/atleta trabalha contra cargas resistivas para melhorar a saúde, a aptidão e o desempenho. Lloyd et al. (2014) explana que as formas de treinamento de resistência incluem o uso de peso corporal, aparelhos de musculação, pesos livres, elásticos e bolas medicinais.

Pesquisas indicaram que várias formas de treinamento resistido podem provocar melhorias significativas em várias vertentes Behringer et al. (2011) apontam benefícios no desempenho da força muscular, Faigenbaum et al. (2005) e Sander et al. (2013) na produção de força, Mikkola et al. (2007) na velocidade de corrida, Thomas, French e Hayes (2009) na agilidade e Behringer et al. (2011) desempenho geral motor na juventude.

Do ponto de vista da saúde, Schwingshandl et al. (1999) indica que o treinamento resistido pode fazer alterações positivas na composição corporal geral, Benson, Torode \& Fiatarone Singh (2008) e Watts, Beye e Siafarikas (2004) demonstram redução na gordura corporal, Shaibi et al. (2006) mostram melhora na sensibilidade à insulina em adolescentes com excesso de peso e Naylor et al. (2008) melhora na função cardíaca em crianças obesas

É importante ressaltar que de acordo com Álvarez-San Emeterio et al. (2011) e Bass (2000) também foi demonstrado que a participação regular em um programa de exercícios adequadamente projetado, incluindo treinamento de resistência, pode melhorar a densidade mineral óssea e melhorar a saú- 
de esquelética e segundo Myer et al. (2011) e Valovich-McLeod et al. (2011) provavelmente reduzir o risco de lesões esportivas em jovens atletas.

Boa parte das modalidades esportivas utilizam elementos da força que é um elemento biomotor, que pode ser associado a resistência, flexibilidade, coordenação e velocidade para gerar variáveis que são de fundamental importância para o desenvolvimento esportivo. A potência é a capacidade de realizar uma ação vigorosa no menor tempo possível (como uma corrida na quadra em busca de um contra-ataque), e se dá pela associação de força + velocidade (Bompa, 2001).

O Handebol tem como necessidade biomotora a força de lançamento, força de aceleração e desaceleração. A força de lançamento é a força empregada em oposição a um implemento, para o handebol uma das principais ações relacionadas a esse elemento é o arremesso da bola. A força de aceleração refere-se a conseguir uma alta velocidade, dependendo da rapidez da contração muscular, como nas situações de contra-ataque. E a força de desaceleração está presente em esportes que o indivíduo deve correr rapidamente e constantemente mudar de direção, observado durante a necessidade repentina de voltar para a defesa, ou a perda da posse de bola entre outras situações. A aceleração e desaceleração exigem muita força nos membros inferiores e superiores, em especial pernas e ombros.

Dentre as diversas modalidades do treinamento de força dentro desse estudo abordaremos dois tipos: o treinamento isométrico e o pliométrico.

O treinamento pliométrico é muito eficaz para o aumento de força e potência, ela utiliza a capacidade de reação do sistema neuromuscular para aumentar os ganhos na potência, partindo de uma contração ex- cêntrica para uma concêntrica, essa característica de potência se dá pelo treinamento do Ciclo Alongamento-Encurtamento (CAE) (Verkhoshanski, 1996). O CAE se fundamenta no conceito de termos propriedades elásticas no músculo e que esse arcabouço é capaz tem capacidade de juntar energia elástica vinda de uma atividade muscular excêntrica (Bosco, et al., 1982). Para uma melhor explicação do CAE, existem os modelos mecânico e neuro físico. No modelo mecânico a energia elástica é produzida nos músculos e tendões e guardada como resultado de um alongamento rápido (Asmussen \& Bonde-Petersen, 1974; Bosco, et al., 1982; Hill, 1970). Essa energia é liberada quando o alongamento é seguido instantaneamente por uma ação de contração muscular. No modelo neuro físico quando um alongamento em alta velocidade é notado nos músculos, uma resposta automática e defendente acontece para evitar uma exorbitância de alongamento muscular, que provocaria uma lesão. Essa resposta é chamada de reflexo de estiramento. O reflexo eleva a atividade dos músculos resignados a uma atividade excêntrica, o que possibilita que ele trabalhe com uma maior demanda de força, resultando em um poderoso efeito de freio e em uma ação concêntrica potente (Komi, 1992; Bosco, et al., 1982). O objetivo do treino de pliometria é diminuir o tempo entre o final da contração muscular excêntrica e o início da contração concêntrica. Este tipo de treino visa conferir a capacidade de treinar padrões de movimento específicos biomecanicamente corretos, fortalecendo o músculo, o tendão e o ligamento de forma mais funcional (Ruivo, Pinheiro \& A. Ruivo, 2018).

A isometria refere-se a uma ação muscular da qual não ocorre alteração total do comprimento do músculo. O treinamento isométrico é realizado comumente como um objeto imóvel ou equipamento com carga maior que a força máxima do indi- 
víduo. Os aumentos na força a partir do treinamento isométrico podem ter relação com a quantidade de ações musculares realizadas, com a duração dessas ações, com o fato de serem ou não máximas e com a frequência de treinamento (Fleck \& Kraemer, 2017). Esse método geralmente é utilizado por profissionais da área da saúde visando a reabilitação, pois durante algumas fases de processo, movimentos articulares não são recomendados tornando esse método uma opção eficaz de treinamento neuromuscular, para esse momento. No âmbito do treinamento físico essa metodologia vem sendo interrogado pois os ganhos de força adquirido com essa metodologia são observados somente na angulação exigida, ou próxima dela (Fleck \& Kraemer, 2017). A isometria também não se apresenta também como uma ação muscular funcional pelo fato de grande parte das ações cotidianas exigirem movimento. Tornando-a, aparentemente, negativa para o desenvolvimento de potência.

Contudo o objetivo desse estudo vai ser analisar os efeitos do treinamento pliométrico e isométrico na força explosiva de membros superior e inferior em jovens atletas do sexo feminino de handebol.

\section{METODOLOGIA}

\section{Casuística}

O presente estudo contou com a participação de 10 atletas do sexo feminino com idade média de 16,1 \pm 0,56 anos, estudantes do ensino médio do Instituto Federal de Roraima, que praticavam handebol a pelo menos três meses. As participantes aceitaram participar da pesquisa assinando o Termo de Assentimento Livre Esclarecido (TALE) e seus pais assinaram o Termo de Consentimento Livre Esclarecido (TCLE).

\section{Protocolos de Treinamento}

As voluntárias foram distribuídas em dois grupos: um com Treinamento Pliométrico (GP) e um com Treinamento Isométrico (GI). Os treinamentos específicos de handebol foram os mesmos em ambos os grupos(3x) sem-45min). Foi realizada uma semana de adaptação aos protocolos isométrico e pliométrico e quatro de treinamento para os grupos, as cargas foram divididas por tempo para ambos os grupo, sendo na adaptação uma serie de 25 segundos, após a adaptação as primeiras duas semanas foram três séries de 25 segundos com o mesmo tempo de intervalo entre elas, na terceira semana o número de séries foi o mesmo, porém, o tempo de execução e descanso aumentou para 30 segundos e na quarta semana houve aumento do tempo de execução e descanso para 35 segundos.

\section{Avaliação de Desempenho da Força Explosiva e Tratamento Estatístico}

A força explosiva de membro inferior foi predita pelo teste de salto horizontal (Gaya e Gaya, 2016), o teste foi feito em dois momentos (AV1 = pré-intervenção e AV2 = após quatro semanas). Na primeira avaliação (AV1) foi aplicado o teste-t de Student para amostras independentes, antes do início do programa de treinamento para avaliar a igualdade entre os grupos (GP e GI) na variável proposta. A variável foi comparada através de análise intragrupo, usou-se o teste-t pareado. O conceito de Effect Size (ES) foi usado no tratamento estatístico dos dados para meIhor entendimento da magnitude do efeito dos treinamentos, nele os valores são divididos entre superficial, pequeno, moderado e grande. O cálculo foi usado para comparar os dois modelos de treinamento propostos no projeto (Rhea, 2004). Na avaliação do ES foram adotados o nível de magnitude para indivíduos e o nível de condicionamento de destreinados, ou seja, indivíduos que treinam a menos de 1 ano. Também foi usa- 
do como referência o índice proposto da PROESP para avaliar o arremesso, a mesma tem índice descrito como fraco, razoável, bom, muito bom e excelência. Todas as análises foram processadas com o software Bioestat ${ }^{\circledR} 5.3$ e para as comparações foi fixado nível crítico menor de 5\% $(p<0,05)$.

\section{Modelo de Periodização}

Modelo Isométrico

\begin{tabular}{lcllll}
\hline EXERCíclO A & ADAPTAÇÃO & \multicolumn{2}{l}{ SEMANA 1 E 2} & \multicolumn{2}{c}{ SEMANA 3 E 4 } \\
\hline Prancha Reta & $1 \times 25^{\prime \prime}$ & $3 \times 25^{\prime \prime}$ & $3 \times 25^{\prime \prime}$ & $3 \times 30^{\prime \prime}$ & $3 \times 35^{\prime \prime}$ \\
\hline Wall Sit & $1 \times 25^{\prime \prime}$ & $3 \times 25^{\prime \prime}$ & $3 \times 25^{\prime \prime}$ & $3 \times 30^{\prime \prime}$ & $3 \times 35^{\prime \prime}$ \\
\hline Flexão Plantar & $1 \times 25^{\prime \prime}$ & $3 \times 25^{\prime \prime}$ & $3 \times 25^{\prime \prime}$ & $3 \times 30^{\prime \prime}$ & $3 \times 35^{\prime \prime}$ \\
\hline EXERCíClO B & & & & & \\
\hline Afundo & $1 \times 25^{\prime \prime}$ & $3 \times 25^{\prime \prime}$ & $3 \times 25^{\prime \prime}$ & $3 \times 30^{\prime \prime}$ & $3 \times 35^{\prime \prime}$ \\
\hline Elevação Pélvica & $1 \times 25^{\prime \prime}$ & $3 \times 25^{\prime \prime}$ & $3 \times 25^{\prime \prime}$ & $3 \times 30^{\prime \prime}$ & $3 \times 35^{\prime \prime}$ \\
\hline Agachamento & $1 \times 25^{\prime \prime}$ & $3 \times 25^{\prime \prime}$ & $3 \times 25^{\prime \prime}$ & $3 \times 30^{\prime \prime}$ & $3 \times 35^{\prime \prime}$ \\
\hline EXERCíclO C & & & & & \\
\hline Prancha Reta & $1 \times 25^{\prime \prime}$ & $3 \times 25^{\prime \prime}$ & $3 \times 25^{\prime \prime}$ & $3 \times 30^{\prime \prime}$ & $3 \times 35^{\prime \prime}$ \\
\hline Wall Sit & $1 \times 25^{\prime \prime}$ & $3 \times 25^{\prime \prime}$ & $3 \times 25^{\prime \prime}$ & $3 \times 30^{\prime \prime}$ & $3 \times 35^{\prime \prime}$ \\
\hline Afundo & $1 \times 25^{\prime \prime}$ & $3 \times 25^{\prime \prime}$ & $3 \times 25^{\prime \prime}$ & $3 \times 30^{\prime \prime}$ & $3 \times 35^{\prime \prime}$ \\
\hline
\end{tabular}

Modelo Pliométrico

\begin{tabular}{|c|c|c|c|c|c|}
\hline \multirow{2}{*}{$\frac{\text { EXERCÍCIO A }}{\text { Remador }}$} & \multirow{2}{*}{$\frac{\text { ADAPTAÇÃO }}{1 \times 25^{\prime \prime}}$} & \multicolumn{2}{|c|}{ SEMANA 1 E 2} & \multicolumn{2}{|c|}{ SEMANA 3 E 4} \\
\hline & & $3 \times 25^{\prime \prime}$ & $3 \times 25^{\prime \prime}$ & $3 \times 30^{\prime \prime}$ & $3 \times 35^{\prime \prime}$ \\
\hline Salto Horizontal & $1 \times 25^{11}$ & $3 \times 25^{\prime \prime}$ & $3 \times 25^{\prime \prime}$ & $3 \times 30^{\prime \prime}$ & $3 \times 35^{\prime \prime}$ \\
\hline Saltito na ponta do pé & $1 \times 25^{11}$ & $3 \times 25^{\prime \prime}$ & $3 \times 25^{\prime \prime}$ & $3 \times 30^{\prime \prime}$ & $3 \times 35^{\prime \prime}$ \\
\hline \multicolumn{6}{|l|}{ EXERCÍCIO B } \\
\hline Jump Squat & $1 \times 25^{11}$ & $3 \times 25^{\prime \prime}$ & $3 \times 25^{\prime \prime}$ & $3 \times 30^{\prime \prime}$ & $3 \times 35^{\prime \prime}$ \\
\hline Subida no Step com saltos & $1 \times 25^{11}$ & $3 \times 25^{\prime \prime}$ & $3 \times 25^{\prime \prime}$ & $3 \times 30^{\prime \prime}$ & $3 \times 35^{\prime \prime}$ \\
\hline Ponte Glútea & $1 \times 25^{11}$ & $3 \times 25^{\prime \prime}$ & $3 \times 25^{\prime \prime}$ & $3 \times 30^{\prime \prime}$ & $3 \times 35^{\prime \prime}$ \\
\hline \multicolumn{6}{|l|}{ EXERCÍCIOC } \\
\hline Jump Squat & $1 \times 25^{11}$ & $3 \times 25^{11}$ & $3 \times 25^{\prime \prime}$ & $3 \times 30^{\prime \prime}$ & $3 \times 35^{\prime \prime}$ \\
\hline Salto Horizontal & $1 \times 25^{11}$ & $3 \times 25^{\prime \prime}$ & $3 \times 25^{11}$ & $3 \times 30^{\prime \prime}$ & $3 \times 35^{\prime \prime}$ \\
\hline Remador & $1 \times 25^{11}$ & $3 \times 25^{11}$ & $3 \times 25^{11}$ & $3 \times 30^{\prime \prime}$ & $3 \times 35^{\prime \prime}$ \\
\hline
\end{tabular}




\section{RESULTADO}

Os dados obtidos na AV1 em relação a membros inferiores apresentaram resultados razoáveis levando em consideração os índices propostos pela PROESP, o grupo pliométrico saltou uma distância de 1,32 $\pm 0,17 m$. Após as quatro semanas propostas esse dado obteve aumento (+3,78\%), resultando em 1,37 $\pm 0,11 \mathrm{~m}$ para o pliométrico, mantendo assim o índice de razoável e a magnitude do efeito foi superficial e $p=0,15$, ou seja, sem diferença estatística. No grupo Isométrico o resultado na primeira avaliação foi de $1,41 \pm 0,12 \mathrm{~m}$ e após a intervenção também teve aumento de 1,56\%, ou seja, 1,43 \pm 0,13m, saindo do índice razoável para bom e tendo uma magnitude de efeito superficial, com $p$ = 0,33, também não apresentando diferença estatística.

Tabela 7

Comparação Entre AV1 e AV2 com magnitude de efeito.

\begin{tabular}{|c|c|c|c|c|c|c|c|c|}
\hline & & AV1 & AV2 & AV2-AV1 & $\Delta \%$ & ES & Magnitude & $\mathbf{P}$ \\
\hline \multicolumn{9}{|c|}{ Membros Inferiores } \\
\hline \multirow[t]{2}{*}{ GP } & $\bar{x}$ & 1,32 & 1,37 & $+0,05$ & $+3,78 \%$ & 0,38 & Superficial & 0,15 \\
\hline & S & 0,17 & 0,11 & & & & & \\
\hline \multirow[t]{2}{*}{ GI } & $\bar{x}$ & 1,41 & 1,43 & $+0,02$ & $+1,56 \%$ & 0,21 & Superficial & 0,33 \\
\hline & $S$ & 0,12 & 0,13 & & & & & \\
\hline
\end{tabular}

Legenda: $A V=$ Avaliação; $\triangle \%$ = Delta Percentual de AV2-AV1; ES = Effect Size; GP = Grupo Pliométrico; GI = Grupo Isométrico; $A V 1$ = Avaliação antes do início do programa; $A V 2$ = Avaliação após 4 semanas de treinamento.

\section{DISCUSSÃO}

Segundo Ruivo, Pinheiro e A. Ruivo (2018) na maioria dos gestos desportivos, os músculos não funcionam de forma unicamente isométrica, concêntrica ou excêntrica, mas funcionam num ciclo muscular semelhante ao da pliometria, de alongamento e encurtamento. Ainda de acordo com Ruivo, Pinheiro e A. Ruivo, op. Cit., sucede-se ao alongamento muscular a fase de encurtamento, ou seja, o ciclo muscular de alongamento e encurtamento caracteriza-se pela produção de uma ação dinâmica concêntrica potente que é imediatamente antecedida de uma ação dinâmica excêntrica do mesmo músculo e é treinável através de exercícios pliométricos.

O crescimento musculoesquelético durante a puberdade, na ausência de adap- tação neuromuscular correspondente, pode facilitar o desenvolvimento de mecanismos articulares anormais e fatores de risco de lesões em jovens adolescentes (Ford et al., 2010). Myer et al. (2006), Myer et al. (2005), Hewett et al. (1996) e Myer et al. (2007) demonstram que programas de treinamento de resistência multifacetados e bem supervisionados reduzem a biomecânica anormal que se manifesta durante a adolescência (ex: aumento do valgo do joelho) e e parecem diminuir as taxas de lesões em atletas do sexo feminino.

Hands (2008), Rogasch et al. (2009), Rosengren, Savelsbergh e van der Kamp (2003) e Faigenbauhn et al. (2011) explanam que o treinamento de resistência utilizado para enriquecer o ambiente de aprendizagem 
motora no início da juventude pode iniciar a adaptação e ajudar as crianças com baixa competência motora a seguirem juntos aos seus pares no controle neuromuscular.

Hewett et al. (1999), Myklebust et al. (2003), Myer et al. (2005), Myer et al. (2011), e Faigenbaum et al. (2011) a participação regular em um programa de treinamento de resistência multifacetado também pode induzir medidas um aumento natural da força muscular, força e coordenação que ocorre com o aumento da idade em adolescentes meninos, que normalmente não são vistos em mulheres, além de reduzir lesões no joelho em atletas adolescentes e mulheres adultas.

Myer et al. (2005) e Hewett et al. (2005) afirmam que o interesse para os profissionais de medicina esportiva, o treinamento resistido com crescimento e desenvolvimento pode induzir o surto neuromuscular desejado, o que pode melhorar o desempenho esportivo e a biomecânica relacionada ao risco de lesões em mulheres jovens. Myer et al. (2005), Ford et al. (2005), Hewett et al. (2006), Kraemer et al. (2001) e Quatman et al. (2006) mostram que os ganhos relativos observados em mulheres podem ser maiores do que em homens, talvez porque os níveis basais de desempenho neuromuscular sejam mais baixos, em média, no sexo feminino.

A pliometria consiste em desenvolver a capacidade de fazer uma rápida transição da ação muscular concêntrica para a excêntrica, isso nos remete ao princípio da especificidade, esse princípio surgiu da ideia de que o treinamento de força ideal deve ser específico. Esse treinamento específico deve conter as habilidades específicas requeridas pela modalidade isso nos lembra que a potência é dada pela aliança de força e velocidade, força essa exercida para fazer o movimento do corpo contra a gravidade e velocidade exigida pelo modelo de treinamento (Bompa, 2001).

Muitos estudos vêm apresentando os exercícios pliométrico como melhorador de desempenho no salto vertical, horizontal, corridas de velocidade e ciclismo (Wilson, et al., 1993, Fatouros, et al., 2000, Potteiger, et al., 1999, Rimmer e Sleivert, 2000). A utilização em atletas treinados de futebol e basquete também apresentou resultados positivos quando se fala em melhora do desempenho (Matavulj, et al., 2001, Diallo, et al., 2001). Flores et al. (2015) analisou a pliometria, com 9 jogadoras de vôlei, apresentando benefícios significativos nas variáveis biomecânicas de salto, em uma periodização de 60 minutos, duas vezes por semana, durante sete semanas. No handebol os dados não apresentaram diferença estatística, mas numericamente apresentaram uma evolução, isso pode ser levado em consideração devido ao baixo número de semanas do treinamento, podendo apresentar melhores diferenças se treinado por mais tempo, como em um estudo que constatou aumento nas fibras de tipo I e II do vasto lateral, após oito semanas de treinamento, o que ocasionou aumento da potência (Potteiger, et al., 1999). Além disso, Rossi \& Brandalize (2007) aponta que a pliometria, além de importante instrumento na reabilitação de lesões, é ainda, efetiva na prevenção destas, pois um bom controle motor atua como um mecanismo protetor capaz de ativar as vias de estabilização reflexas, mecanismos de antecipação, ocasionando uma resposta motora mais veloz diante de forças ou traumas inesperados.

Brunner et al. (2019) aponta que os programas de exercícios com membros inferiores multicomponentes, incluindo pliometria, foram eficazes na prevenção de lesões nos membros inferiores, joelho, ligamento cruzado anterior e tornozelo nos esportes coletivos de futebol, floorball, futebol americano e australiano, basquete, handebol e vôlei para todas as idades e sexo. 
A pliometria é, portanto, uma forma de se obter força explosiva e melhorar a propriocepção ao mesmo tempo.

O treinamento isométrico é quando o musculo é ativado e produz força, mas não apresenta movimento (Fleck \& Kraemer, 2017). Isso pode ser mantido com um peso estacionário ou uma carga maior que a suportável, visando o princípio da especificidade, se o indivíduo treina de maneira isométrica e faz um teste estático um grande aumento de força pode ser encontrado, isso é o princípio da especificidade. O ganho de força semanal no treinamento isométrico é de 5\%, porém esse ganho é no ângulo treinado (Hettinger \& Muller, 1953). A isometria pode provocar ganhos de força estático que podem ser substanciais e variáveis ao longo do período de treinamento de curta duração (Fleck \& Schutt, 1983). Analisando os resultados obtidos nesse trabalho, houve um relativo aumento na potência dos indivíduos $(+4,56 \%$ para membro superior e $+1,56 \%$ para membro inferior), apesar da proposta de treinamento estático, esse acréscimo na potência pode-se dar pelo aumento da força proposto pelo método. Essa ideia contrapõe-se a (Teixeira \& Guedes, 2016) que afirmam que a isometria não apresenta característica funcional para o esporte, contudo a associação desse treino com o da modalidade específica pode ter ocasionado os ganhos pois em um estudo com universitárias apontou que o treino específico de corrida não atrapalhou no desempenho da força de membros superiores e tronco (Raddi, et al., 2008), isso nos leva a adotar o uso do treinamento isométrico concomitante ao da modalidade específica como mais uma opção na periodização. Exercícios para estabilidade do core são fundamentais para todo tipo de atletas, os músculos do core são fortemente trabalhados com a força estática devido aos ganhos de força no ângulo trabalhado (Contreras, 2016). Um estudo que apresentou aumento da potência de lutadores de judô com o treinamento isométrico (Campos, et al., 2018), outro estudo o treinamento muscular isométrico apresentou maior ganho na taxa de força em relação ao isocinético concêntrico (Corvino, et al., 2009).

A dissertação de mestrado de Soares (2018) que comparou diversas variáveis, dentre elas a potência de membros inferiores através do teste de salto horizontal mostrou que houve, após 8 semanas da aplicação dos métodos de treinamento, um aumento na distância nos três grupos analisados, sendo $3,0 \%$ no grupo pliométrico, 10,6\% no grupo isométrico e 5,5\% no grupo isotônico.

Pouco se aborda sobre a relação isometria e potência, porém deve-se começar a analisar a adição dessa modalidade de treino somado a outras, visto que a ciência aponta um exponencial ganho de força, como supracitado, então combinar momentos de isometria seguido de momentos de velocidade, pliometria, agilidade ou características específicas da modalidade pode apresentar mudanças significativas.

\section{CONCLUSÃO}

A pesquisa conclui que quatro semanas de treinamento de ambos os métodos não são suficientes para apresentar diferença estatística significativa na força explosiva de membro inferior, mas apresentou diferenças numéricas, e magnitude de efeito superficial para esses membros. Com esses resultados abre-se a possibilidade de analisar os métodos com maior tempo de duração e fazer um experimento trabalhando as duas metodologias de maneira concomitante. As propostas também devem ser estudadas para trabalho de prevenção de lesões e melhora de outras valências física. 


\section{BIBLIOGRAFIA}

Álvarez-San Emeterio, C., Antuñano, N. P. G., López-Sobaler, A. M., et al. (2011). Effect of strength training and the practice of Alpine skiing on bone mass density, growth, body composition, and the strength and power of the legs of adolescent skiers. The Journal of Strength \& Conditioning Research, 25(10), 2879-2890.

Asmussen, E. e Bonde-Petersen, F. (1974). Storage of elastic energy in skeletal muscle in man. Acta Physiologica Scandinavica. 97(3), 385-392. DOI: 10.1117/j.1748-1716.1974.tb05693.x

Bass, S. L. (2000). The prepubertal years: a uniquely opportune stage of growth when the skeleton is most responsive to exercise?. Sports Medicine 30(2), 73-78. DOI: 10.2165/00007256200030020-00001

Behringer, M., Vom Heede, A., Matthews, M., et al. (2011). Effects of strength training on motor performance skills in children and adolescents: a metaanalysis. Pediatric Exercise Science, 23(2), 186-206. DOI: 10.1123/pes.23.2.186
Bosco, C., et al. (1982). Neuromuscular function and mechanical efficiency of human leg extensor during jump exercises. Acta Physiologica Scandinavica, 174(4), 543-50. DOI: 10.1171/ j.1748-1716.1982.tb07022.x

Brunner, R., Friesenbichler, B., Casartelli, N. C., et al. (2019). Effectiveness of multicomponent lower extremity injury prevention programmes in team-sport athletes: an umbrella review. British Journal of Sports Medicine, 53(5), 282- 288. DOI: 10.1136/bjsports-2017-098944

Campos, B. T., et al. (2018). Exercício preparatório de força isométrica aumenta o desempenho de potência muscular de judocas em testes específicos e não específicos. Journal of Physical Education, 29(1), e-2910. DOI: https:// doi.org/10.4025/jphyseduc.v29i1.2910

Contreras, B. (2016). Anatomia do Treinamento de força. São Paulo: Manole.

Corvino, R. B., et al. (2009). Taxa de desenvolvimento de força em diferentes velocidades de contrações musculares. Revista Brasileira de Medicina do Esporte, 15(6), 428-431. DOI: http://dx.doi.org/10.1590/S151786922009000700005

Diallo, O., et al. (2001). Effects of plyometric training followed by a reduced training programme on physical performance in prepubescent soccer players. Journal of Sports Medicine and Physical Fitness, 47(3), 342-348.

Bosco, C., et al. (1982). Combined effect of elastic energy and myoelectrical potentiation during stretch-shortening cycle exercise. Acta Physiologica Scandinavica, 714(4), 554-565. DOI: 10.1111/j.1748-1716.1982.tb07024.x
Eleno, T. G., Barela, J. A. \& Kokubun, E. (2002). Tipos de esforço e qualidades físicas do handebol. Revista Brasileira de Ciências do Esporte, 24(1), 83-98. 
Faigenbaum, A. D., Farrell, A., Fabiano, M., et al. (2011). Effects of integrative neuromuscular training on fitness performance in children. Pediatric exercise science, 23(4), 573-584. DOI: 10.1123/ pes.23.4.573

Faigenbaum, A. D., Milliken, L., Moulton, L., \& Westcott, W. L. (2005). Early muscular fitness adaptations in children in response to two different resistance training regimens. Pediatric Exercise Science, 77(3), 237-248. DOl: https:// doi.org/10.1123/pes.17.3.237

Fatouros, I. G., et al. (2000). Evaluation of PIyometric Exercise Training, Weight Training, and Their Combination on Vertical Jumping Performance and Leg Strength. Journal of Strength and Conditioning Research, 14(4), 470-476. DOI: 10.1519/00124278200011000-00016

Fleck, S. J. \& Schutt, J. R. (1983). Types of strength training. The Orthopedic clinics of North America, 14(2),449-458.

Fleck, S.J. \& Kraemer, J.W. (2017). Fundamentos do treinamento de força muscular. Porto Alegre: Artmed.

Flores, A., Araya, S., Guzmán, R. \& Montecinos, R. (2015). Efecto de un programa de entrenamiento pliométrico sobre la biomecánica de salto en mujeres voleibolistas juveniles. Revista Ciencias de la Actividad Física UCM. 16 (1), 37-44. Recuperado de http://revistacaf.ucm.cl/article/view/70

Ford, K.R., Shapiro, R., Myer, G.D., et al. (2010). Longitudinal sex differences during landing in knee abduction in young athletes. Medicine \& Science in Sports \& Exercise. 42(10),1923-1931. DOI: 10.1249/MSS.0b013e3181dc99b1
Ford, K. R., Myer, G. D., Smith, R. L., et al. (2005). Use of an overhead goal alters vertical jump performance and biomechanics. The Journal of Strength \& Conditioning Research, 19(2), 394399. DOI: 10.1519/15834.1

Gaya, A. \& Gaya, A. (2016). Projeto esporte Brasil proesp - Bateria de Medidas e Testes Somatomotores do Proesp. Universidade Federal do Rio Grande do Sul. [Online] https://www.ufrgs.br/ proesp/

Hands, B. (2008). Changes in motor skill and fitness measures among children with high and low motor competence: A five-year longitudinal study. Journal of Science and Medicine in Sport, 17(2), 155-162. DOI: 10.1016/j. jsams.2007.02.012

Hettinger, T. \& Müller, E. A. (1953). Muskelleistung und muskeltraining. Arbeitsphysiologie, 15(2)111-126.

Hewett, T. E., Lindenfeld, T. N., Riccobene, J. V., et al. (1999). The effect of neuromuscular training on the incidence of knee injury in female athletes. A prospective study. The American Journal of Sports Medicine, 27(6), 699-706. DOI: 10.1177/03635465990270060301 (2005). Biomechanical measures of neuromuscular control and valgus loading of the knee predict anterior cruciate ligament injury risk in female athletes: a prospective study. The American journal of sports medicine, 33(4), 492-501. DOI: $10.1177 / 0363546504269591$ 
Hewett, T. E., Myer, G. D., Ford, K. R., et al. (2006). Preparticipation physical examination using a box drop vertical jump test in young athletes: the effects of puberty and sex. Clinical Journal of Sport Medicine, 76(4), 298-304. DOI: 10.1097/00042752200607000-00003

Hewett, T. E., Stroupe, A. L., Nance, T. A., \& Noyes, F. R. (1996). Plyometric training in female athletes. Decreased impact forces and increased hamstring torques. The American journal of sports medicine, 24(6), 765-773. DOI: $10.1177 / 036354659602400611$

Hill, A. V. (1970). First and last experiments in muscle mechanics. New York: Cambridge University Press.

Kokubun, E., \& Daniel, J. F. (1992). Relações entre a intensidade e duração das atividades em partida de basquetebol com as capacidades aeróbica e anaeróbica: estudo pelo lactato sangüíneo. Revista Paulista De Educação Física, 6(2), 37-46. DOI: https:// doi.org/10.11606/issn.2594-5904 rpef.1992.138070

Komi, P. V. (1992). Strength and power in sport. Oxford: Blackwell.

Kraemer, W. J., Keuning, M., Ratamess, N. A., et al. (2001). Resistance training combined with bench-step aerobics enhances women's health profile. Medicine \& Science in Sports \& Exercise, 33(2), 259-269. DOI: 10.1097/00005768200102000-00015

Lloyd, R. S., Faigenbaum, A. D., Stone, M. H., Oliver, J. L., Jeffreys, I., Moody, J. A., .. \& Herrington, L. (2014). Position statement on youth resistance training: the 2014 International Consensus. British Journal of Sports Medicine, 48(7), 498-505. DOI: http://dx.doi.org/10.1136/ bjsports-2013-092952
Martini, K. (1980). Andebol: técnica, tática, metodologia. Mem Martins: Publicações Europa-América.

Matavulj, D., et al. (2001). Effects of plyometric training on jumping performance in junior basketball players. Journal of sports medicine and physical fitness 47(2), 159-164. Recuperado de https://www.ncbi.nlm.nih.gov/pubmed/11447356

Mikkola, J., Rusko, H., Nummela, A., et al. (2007). Concurrent endurance and explosive type strength training improves neuromuscular and anaerobic characteristics in young distance runners. International journal of sports medicine, 28(07), 602-611. DOI: 10.1055/s-2007-964849

Myer, G. D., Faigenbaum, A. D., Chu, D. A., et al. (2011). Integrative training for children and adolescents: techniques and practices for reducing sportsrelated injuries and enhancing athletic performance. The Physician and sportsmedicine, 39(1), 74-84. DOI: 10.3810/psm.2011.02.1854

Myer, G. D., Faigenbaum, A. D., Ford, K R., et al. (2011). When to initiate integrative neuromuscular training to reduce sports-related injuries in youth? Current sports medicine reports, 10(3), 155. DOI: 10.1249/ JSR.0b013e31821b1442

Myer, G. D., Ford, K. R., Brent, J. L., \& Hewett, T. E. (2006). The effects of plyometric vs. dynamic stabilization and balance training on power, balance, and landing force in female athletes. Journal of strength and conditioning research, 20(2), 345. DOI: 10.1519/R17955.1 
Myer, G. D., Ford, K. R., Brent, J. L., \& Hewett, T. E. (2007). Differential neuromuscular training effects on $A C L$ injury risk factors in" high-risk" versus" low-risk" athletes. BMC musculoskeletal disorders, 8(1), 39. DOI: 10.1186/1471-2474-839

Myer, G. D., Ford, K. R., Palumbo, O. P., \& Hewett, T. E. (2005). Neuromuscular training improves performance and lower-extremity biomechanics in female athletes. The Journal of Strength \& Conditioning Research, 19(1), 51-60. DOI: 10.1519/13643.1

Myklebust, G., Engebretsen, L., Brækken, I., et al. (2003). Prevention of anterior cruciate ligament injuries in female team handball players: a prospective intervention study over three seasons. Clinical journal of sport medicine, 13(2), 71-78. DOI: 10.1097/00042752200303000-00002

Naylor, L. H., Watts, K., Sharpe, J. A., et al. (2008). Resistance training and diastolic myocardial tissue velocities in obese children. Medicine and science in sports and exercise, 40(12), 2027-2032. DOI: 10.1249/ MSS.0b013e318182a9e0

Potteiger, J. A., et al. (1999). Muscle power and fiber characteristics following 8 weeks of plyometric training. Journal of Strength and Conditioning Research. 13(3), 275-279,

Quatman, C. E., Ford, K. R., Myer, G. D., \& Hewett, T. E. (2006). Maturation leads to gender differences in landing force and vertical jump performance: a longitudinal study. The American journal of sports medicine, 34(5), 806-813. DOI: 10.1177/0363546505281916
Raddi, L.L.O., et al. (2008). Treino de corrida não interfere no desempenho de força de membros superiores. Revista Brasileira de Medicina do Esporte. 14(6), 544-547. DOI: 10.1590/s151786922008000600014

Rhea, M.R. (2004). Determining the magnitude of treatment effects in strength training research through the use of the effect size. Journal of Strength and Conditioning Research, 18(4), 918-920. DOI: 10.1519/14403.1

Rimmer, E., \& Sleivert, G. (2000). Effects of a plyometrics intervention program on sprint performance. The Journal of Strength \& Conditioning Research. 74(3), 295-301.

Rogasch, N. C., Dartnall, T. J., Cirillo, J., et al. (2009). Corticomotor plasticity and learning of a ballistic thumb training task are diminished in older adults. Journal of applied physiology, 107(6), 1874-1883. DOI: 10.1152/japplphysiol.00443.2009

Rosengren, K. S., Savelsbergh, G. J., \& van der Kamp, J. (2003). Development and learning: a TASC-based perspective of the acquisition of perceptual-motor behaviors. Infant Behavior and Development, 26(4), 473-494. DOI: https:// doi.org/10.1016/j.infbeh.2003.08.001

Rossi, L. P., \& Brandalize, M. (2007). Pliometria aplicada à reabilitação de atletas. Revista Salus, 7(1). Recuperado de https://revistas.unicentro.br/index. php/salus/article/view/674

Ruivo, R., Pinheiro, V., \& Ruivo, J. (2018). Prevenção de Lesões no Futebol: Bases Científicas e Aplicabilidade. Revista de Medicina Desportiva informa, 9(2), 16-19. DOI: https://doi.org/10.23911/ Vol.9lss.2Prevencao_lesao 
Sander, A., Keiner, M., Wirth, K., \& Schmidtbleicher, D. (2013). Influence of a 2-year strength training programme on power performance in elite youth soccer players. European journal of sport science, 13(5), 445451. DOI: https://doi.org/10.23917/ Vol.9lss.2Prevencao_lesao

Schwingshandl, J., Sudi, K., Eibl, B., et al. (1999). Effect of an individualised training programme during weight reduction on body composition: a randomised trial. Archives of disease in childhood, 87(5), 426-428. DOI: http:// dx.doi.org/10.1136/adc.81.5.426

Shaibi, G. Q., Cruz, M. L., Ball, G. D. et al (2006). Effects of resistance training on insulin sensitivity in overweight Latino adolescent males. Medicine and science in sports and exercise, 38(7), 1208-1215. DOI: 10.1249/01. mss.0000227304.88406.0f

Soares, M. D. S. (2018). Gerenciamento dos treinamentos isotônico, isométrico e pliométrico para identificar a variação da força muscular dos membros inferiores de atletas amadores de futebol americano. (Dissertação de Mestrado). Universidade do Estado de São Paulo - UNESP - Guaratinguetá, SP, Brasil.

Teixeira, C. V. L. S. \& Guedes Jr, D. P. (2016). Musculação Funcional: ampliando os limites da prescrição tradicional. São Paulo: Phorte.

Thomas, K., French, D., \& Hayes, P. R. (2009). The effect of two plyometric training techniques on muscular power and agility in youth soccer players. The Journal of Strength \& Conditioning Research, 23(1), 332-335. doi: 10.1519/ Jsc.0b013e318183a01a
Valovich McLeod, T. C., Decoster, L. C., Loud, K. J., et al. (2011). National Athletic Trainers' Association position statement: prevention of pediatric overuse injuries. Journal of athletic training, 46(2), 206-220. DOI: https://doi. org/10.4085/1062-6050-46.2.206

Verkhoshanski, I.V. (1996). Força - Treinamento da potência muscular, método de choque (1a Ed.). Londrina: CID Centro de Informações Desportivas.

Watts, K., Beye, P., Siafarikas, A., et al. (2004). Exercise training normalizes vascular dysfunction and improves central adiposity in obese adolescents. Journal of the American College of Cardiology, 43(10), 1823-1827. DOI: 10.1016/j.jacc.2004.01.032

Wilson, G.J., et al. (1993). The optimal training load for the development of dynamic athletic performance. Medicine and Science in Sports and Exercise. 25(11), 1279-1286. DOI: 10.1249/00005768199311000-00013

\section{Dirección para correspondencia}

João Victor da Costa Alecrim

Graduado em Educação Física

Laboratório de Biociência e Motricidade

Humana de Roraima.

Boa Vista, Brasil.

Rua amapa, 709, Bairro dos estados, CEP: 69.305-520, Boa Vista - RR. Brasil. ORCID iD: https://orcid.org/0000-0002-7449-0116

Contacto:

joaovictoralecrim73@gmail.com

Recibido: 07-05-2019

Aceptado: 09-12-2019 